Reprod. Nutr. Dévelop., 1983, 23 (6), 1029-1042.

\title{
Effets sur la digestion et le métabolisme des vaches laitières d'infusions d'acides gras volatils dans le rumen et de caséinate dans le duodénum. II. - Métabolisme général et mammaire
}

\section{H. RULQUIN}

avec la collaboration technique de Jeanne FLÉCHET, Renée LEFAIVRE, A. OLLIER et Claire SORNET

Station de Recherches sur la Vache laitière, I.N.R.A., Saint-Gilles, 35590, L'Hermitage, France.

Summary. Effects of ruminal infusion of volatile fatty acids and duodenal infusion of caseinate on digestion and metabolism in the dairy cow. II. - General and mammary metabolisms.

In a previous study (Rulquin, 1982) using a latin square and 4 dairy cows, ruminal infusions (2.4 or 23.8 moles/day) of a mixture of propionic acid-rich volatile fatty acids (VFA) combined with duodenal infusions of caseinate $(0$ or $500 \mathrm{~g} /$ day) changed milk secretion and its constituents.

The aim of the present work was to try to determine the mechanism of these effects by measuring the mammary arterio-venous differences ( $A-V$ differences) of the precursors of some milk constituents.

1) VFA infusion caused the A-V differences of all the energetic nutrients to decrease by 15 to $38 \%$ as the result of a reduction either in the arterial level of acetate and $\beta$-hydroxybutyrate $(20$ and $29 \%)$ or in the extraction rate of glucose and triglycerides (19 and $27 \%$ ) (table 1). The arterial levels of all essential amino acids were decreased on an average by $13 \%$ (mainly those of branched amino acids : -21 to $-24 \%$ ), while their A$\checkmark$ differences were unchanged (table 2).

2) The infusion of caseinate mainly caused arterial levels and A-V differences of all essential amino acids to increase (an average of 60 and $55 \%$ ), and especially those of branched amino acids (104 and $57 \%$ ). According to their respective variations in the artery and duodenum, methionine, threonine, phenylalanine, histidine and lysine seemed to be, in this order, the most limiting (fig. 3).

3) The associative effect between energy and nitrogen that caused wide variations in milk secretion constituents produced no significant change in A-V differences; it only affected arterial levels of urea, isoleucine, valine, phenylalanine and threonine. Of the limiting amino acids mentioned previously, histidine and lysine seemed to be limiting only with the low energy level (fig. 4).

These effects have been discussed in relation with putative hormonal changes caused by infusions and possibly orienting the utilization of nutrients towards extra-mammary tissues or modifying the effectiveness of their utilization by the udder. 
Introduction.

Un accroissement de la sécrétion du lait et de ses constituants a été obtenu chez des vaches laitières par une augmentation des apports d'acides aminés (infusion duodénale de caséinate), mais pas par celle des apports énergétiques (infusion ruminale d'acide propionique) (Rulquin, 1982). Des effets similaires avaient été obtenus au cours d'essais d'infusion (Storry et Rook, 1965 ; revue de Clark, 1975) ou d'alimentation avec des régimes riches en aliments concentrés (Annison, Bickerstaffe et Linzell, 1974). De plus, une interaction très importante entre le niveau des apports énergétiques et azotés a été observée comme dans l'essai d'alimentation de Gordon et Forbes (1970).

Pour comprendre les mécanismes de ces effets, l'étude détaillée de la digestion dans les différents compartiments du tube digestif devait être complétée par celle du métabolisme mammaire. En effet, des modifications du métabolisme général et/ou mammaire ont été notées pour l'énergie par Annison, Bickerstaffe et Linzell (1974) et pour l'azote par Clark et al. (1977). Des mesures des différences artério-veineuses mammaires des principaux nutriments ont donc été effectuées sur ces animaux partiellement nourris par infusions.

\section{Matériel et méthodes.}

Deux niveaux d'apports énergétiques (85 ou $110 \%$ des besoins) et deux niveaux d'apports azotés (70 ou $100 \%$ des besoins) en combinaison factorielle ont été comparés sur 4 vaches en lactation suivant un carré latin comportant des périodes de 4 semaines. Les aliments apportés couvraient $80 \%$ des besoins énergétiques et $70 \%$ des besoins azotés. Le complément était obtenu par infusion intra-ruminale d'AGV $(2,4$ ou 23,8 moles/j) et par infusion intra-duodénale de caséinate de sodium 10 ou $500 \mathrm{~g} / \mathrm{j}$ ). Le mélange d'acides gras volatils (AGV) comprenait en moles : $32 \%$ d'acide acétique, $56 \%$ de propionique, $12 \%$ de butyrique. Les détails concernant les animaux, leur alimentation et la réalisation des infusions correspondent à ceux qui ont été rapportés précédemment (Rulquin, 1982).

Outre les mesures pertant sur l'ingestion, la production de lait et la digestion dans le rumen et dans l'intestin (Rulquin, 1982), des séries de différences artérioveineuses mammaires des principaux précurseurs des constituants du lait ont été mesurées pendant deux jours consécutifs en quatrième semaine de chaque période. Les prélèvements de sang ont été effectués simultanément sur la carotide et sur la veine abdominale sous-cutanée à $7 \mathrm{~h}, 8 \mathrm{~h} 30,10 \mathrm{~h}, 13 \mathrm{~h}$ et $16 \mathrm{~h}$, selon la technique décrite précédemment (Rulquin, 1981).

A chaque période et pour chaque vache, un échantillon regroupant les dix points de prélèvements a été réalisé pour analyser les acides aminés libres du sang selon la technique de Moore et Stein, modifiée par Pawlak et Pion (1968). Après centrifugation, une partie du plasma obtenu a été déféquée pour l'analyse du glucose, de l'acétate, du $\beta$-hydroxybutyrate et de l'urée (Rulquin, 1981). Excepté pour les prélèvements de $8 \mathrm{~h} 30$, le reste du plasma a été utilisé pour le dosage 
des acides gras non estérifiés (AGNE) selon la méthode d'Antonis (1965), et celui du glycérol total par méthode enzymatique (Bas, non publié).

Une des vaches ayant dû être retirée de l'expérience à la suite d'un coma acidosique, l'analyse statistique des résultats a été effectuée suivant la méthode des carrés de Youden (Cochran et Cox, 1968) à partir de la moyenne des données recueillies par vache et par période, c'est-à-dire une seule mesure pour les acides aminés, la moyenne de 18 mesures pour les AGNE et le glycérol, la moyenne de 20 mesures pour les autres métabolites. Les résultats présentés correspondent aux moyennes ajustées.

\section{Résultats.}

Effets du niveau énergétique.

- Paramètres du métabolisme énergétique. - L'élévation du niveau énergétique par infusion d'AGV dans le rumen conduit à une diminution importante et significative des taux artériels moyens d'acétate $(-20 \%)$, de $\beta$-hydroxybutyrate $(-29 \%)$ et de leurs différences artério-veineuses (différences $A-V)$ respectives $(-25$ et $-38 \%)$, sans modification significative de leur pourcentage d'extraction mammaire (tabl. 1).

TABLE 1

Effets des niveaux énergétiques et azotés sur les paramètres du métabolisme énergétique

$\begin{array}{lllll}\text { Niveau énergétique } & \text { Niveau azoté } & \begin{array}{c}\text { Ecart-type } \\ \text { des } \\ \text { différences } \\ \text { entre } \\ \text { Bas }\end{array} \text { Haut } & \text { Bas Haut Hnes }\end{array}$

Taux artérie/s (A)

- acétate $(\mathrm{mg} / 100 \mathrm{ml}) \ldots \ldots \ldots \ldots$.

- $\beta$-hydroxybutyrate $(\mathrm{mg} / 100 \mathrm{ml}) \ldots$

- glucose $(\mathrm{mg} / 100 \mathrm{ml}) \ldots \ldots \ldots \ldots$

\section{9,37}

8,51

66,85

- glycérol ( $\mathrm{mg} / \mathrm{l}$ )

4,89

- AGNE $(\mu \mathrm{eq} / 1) \ldots \ldots \ldots \ldots \ldots \ldots$.

$7,54^{* *}$
$6,02^{*}$
$70,2^{*}$
5,11
80,0

\section{8,10}

7,21

67,6

5,08

89,0
$8,81+$

7,32

69,5

4,91

76,2
0,33

0,69

0,95

0,22

9,25

Différences artério-veineuses (A-V)

- acétate $(\mathrm{mg} / 100 \mathrm{ml}) \ldots \ldots \ldots \ldots$. . .

- $\beta$-hydroxybutyrate $(\mathrm{mg} / 100 \mathrm{ml}) \ldots$

- glucose $(\mathrm{mg} / 100 \mathrm{ml}) \ldots \ldots \ldots \ldots$

- glycérol (mg/l)

$\begin{array}{cc}6,47 & 4,83^{* *} \\ 3,87 & 2,39^{*} \\ 12,3 & 10,5+ \\ 2,00 & 1,52 \\ -16,6 & -31,2\end{array}$

\section{5,36}

3,23

10,8

1,55

$\begin{array}{ll}-24,6 & -23,2\end{array}$

$5,94+$

0,21

3,03

0,35

12,0

0,79

- AGNE ( $\mu$ eq/l)

$\ldots \ldots \ldots \ldots \ldots$

Pourcentages d'extraction $\frac{(A-V)}{A} \times(100)$

- acétate ................

- $\beta$-hydroxybutyrate ............

- glucose . . . . ...............

68,9

45,5

- glycérol

18,3

$$
\begin{array}{r}
64,4 \\
40,0
\end{array}
$$

66,2

67,2

0,21

39,0

$39,2+$

3,59

AGNE : acides gras non estérifiés. Degrés de signification : $P<0,1:+; P<0,05:{ }^{*}$; $\mathrm{P}<0,01:{ }^{*}$. 
Au cours de la journée, cet effet est maximum durant les trois heures qui suivent la distribution des aliments et se maintient jusqu'à la distribution suivante à un niveau plus élevé pour le $\beta$-hydroxybutyrate que pour l'acétate (fig. 1).

Les taux artériels moyens de glucose varient dans le même sens que le niveau énergétique $(+5 \%)$, alors que les différences $A-V$ et les extractions mammaires varient en sens opposé (-14 et $-19 \%$ ) (tabl. 1). L'effet sur les taux artériels est relativement constant au cours de la journée, mais ne devient significatif que $5 \mathrm{~h}$ après la distribution des aliments (fig. 1). Par contre, l'effet sur les différences $A-V$ est important et significatif juste avant la distribution et durant les $3 \mathrm{~h}$ qui suivent (fig. 1).

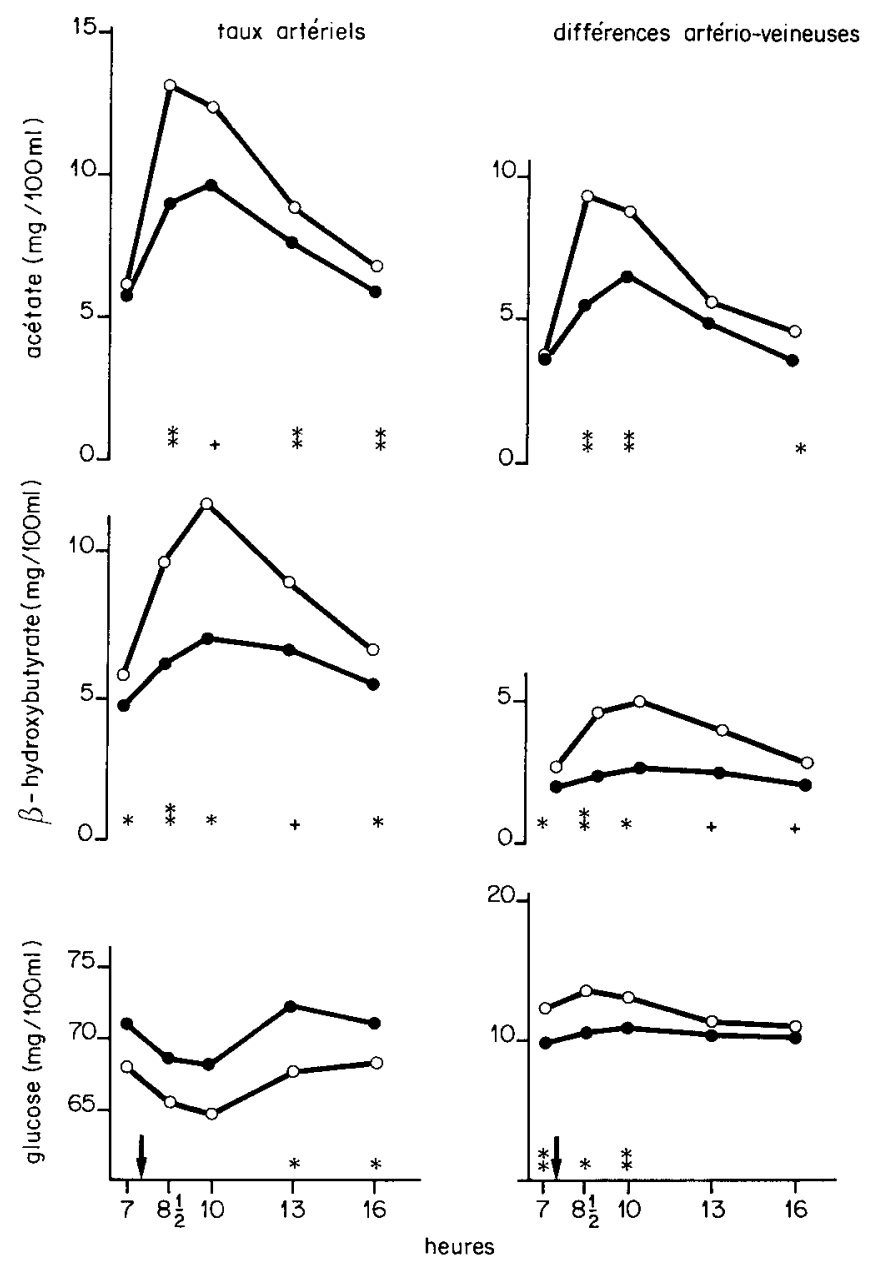

FIG. 1. - Effets du niveau énergétique sur les évolutions des paramètres du métabolisme énergétique.

- : niveau Haut ; $O$ : niveau Bas ; 1 : distribution des aliments ; $P<0,1$ : $+\mathrm{P}<0,05$ : ${ }^{*}$; $\mathrm{P}<0,01:{ }^{* *}$. 
Les taux artériels et les différences A-V moyens de glycérol et d'AGNE ne sont pas significativement modifiés par les variations du niveau énergétique. Cependant, le pourcentage d'extraction du glycérol est significativement $(P<0,1)$ plus faible avec le niveau énergétique haut (tabl. 1). Avant la distribution des aliments, les taux artériels d'AGNE sont plus élevés pour le niveau énergétique bas que pour le niveau haut (108 vs $76 \mu \mathrm{eq} / \mathrm{I}$ ), mais de façon non significative.

- Paramètres du métabolisme azoté. - Les taux artériels d'urée et de l'ensemble des acides aminés libres sanguins diminuent significativement ( $P<0,05$ et $P<0,1$ ) avec l'accroissement du niveau énergétique (tabl. 2). La baisse de l'aminoacidémie est due principalement aux acides aminés indispensables (arginine et tyrosine exceptées) et plus particulièrement aux acides aminés ramifiés $(-21$ à $24 \%$ ) et à un moindre degré à la phénylalanine et la thréonine ( - 6 et $-13 \%$ ) (tabl. 2). Parmi les acides aminés non indispensables, seule la proline est significativement plus faible $(-8 \%)$ avec le niveau énergétique haut $(P<0,1)$.

Les différences $A-V$ des acides aminés indispensables sont pratiquement identiques entre les deux niveaux énergétiques. Celles des acides aminés non indispensables sont supérieures mais non significativement avec le niveau haut (tabl. 2).

\section{Effets du niveau azoté.}

- Paramètres du métabolisme énergétique. - Les modifications des valeurs moyennes des paramètres du métabolisme énergétique dues à l'infusion de caséinate sont relativement faibles et seules les augmentations des taux artériels et des différences $A-V$ d'acétate (9 et $11 \%$ ) et celles du pourcentage d'extraction du glycérol $(+40 \%)$ sont significatives $(P<0,1)$ (tabl. 1$)$.

L'effet sur l'acétate est surtout sensible dans les $3 \mathrm{~h}$ qui suivent la distribution des aliments (fig. 2). Bien que les différences $A-V$ de glucose ne soient pas significativement différentes sur la moyenne des prélèvements, elles sont supérieures avec le niveau haut juste avant la distribution d'aliment et durant les $3 \mathrm{~h}$ qui suivent $(P<0,05)$ (fig. 2).

- Paramètres du métabolisme azoté. - L'élévation du niveau azoté a conduit à des augmentations importantes et significatives des taux artériels d'urée $(+136 \%$ ) et de l'aminoacidémie libre $(+30 \%)$ (tabl. 2). Cet effet est beaucoup plus important pour les acides aminés indispensables $(+60 \%)$ que pour les non indispensables $(+7 \%)$.

Les taux artériels de tous les acides aminés indispensables s'accroissent significativement de : 15 à $25 \%$ pour la méthionine, la thréonine et la phénylalanine, 30 à $47 \%$ pour la tyrosine, la lysine et l'histidine, 80 à $128 \%$ pour l'arginine et les acides aminés ramifiés (tabl. 2). Parmi les acides aminés non indispensables, seules la diminution du taux de glycine et les augmentations de ceux d'alanine, d'ornithine et de proline sont significatives (tabl. 2).

Les différences $A-V$ des acides aminés indispensables varient dans le même sens que les taux artériels, mais seules les augmentations de celles des acides 


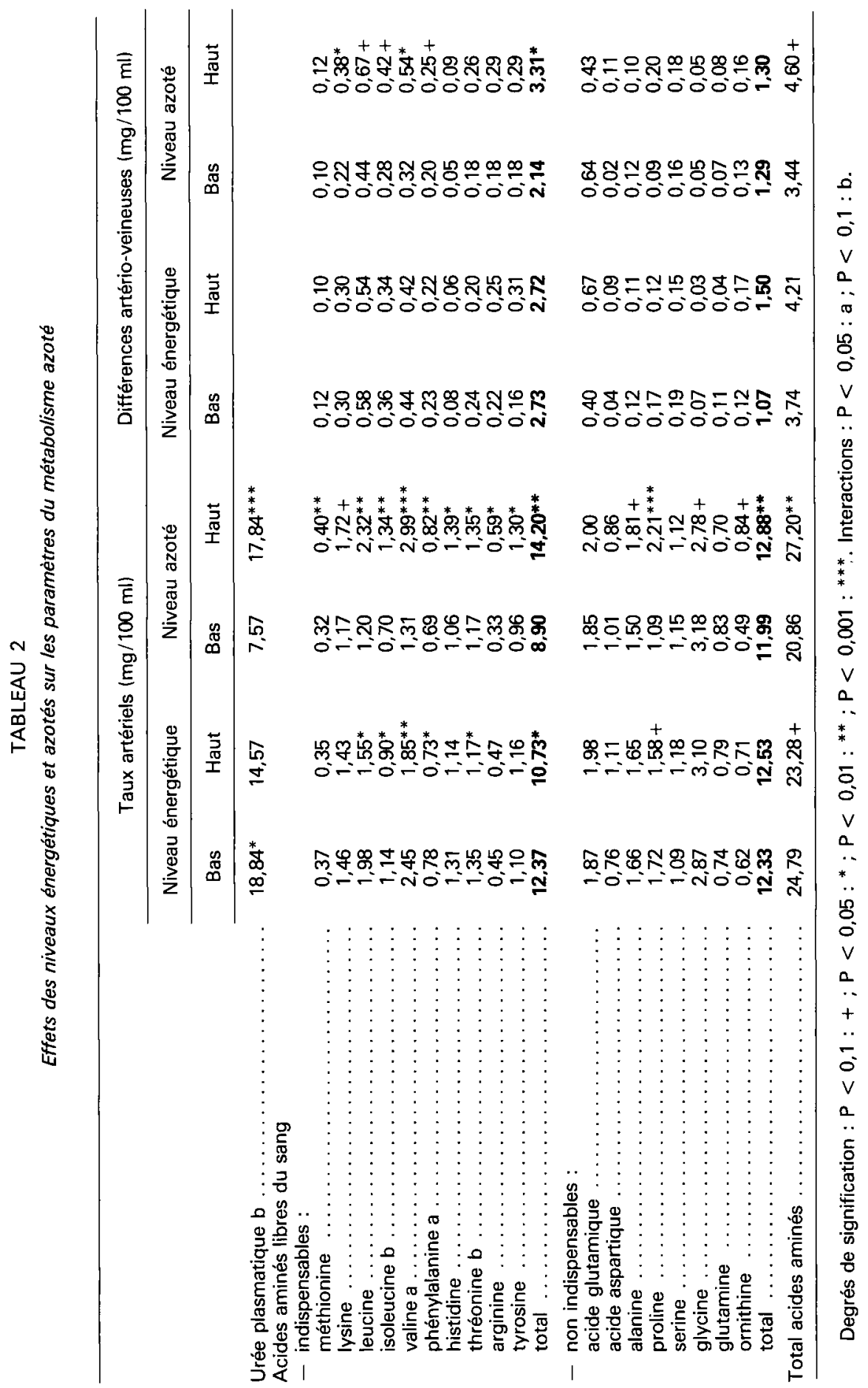



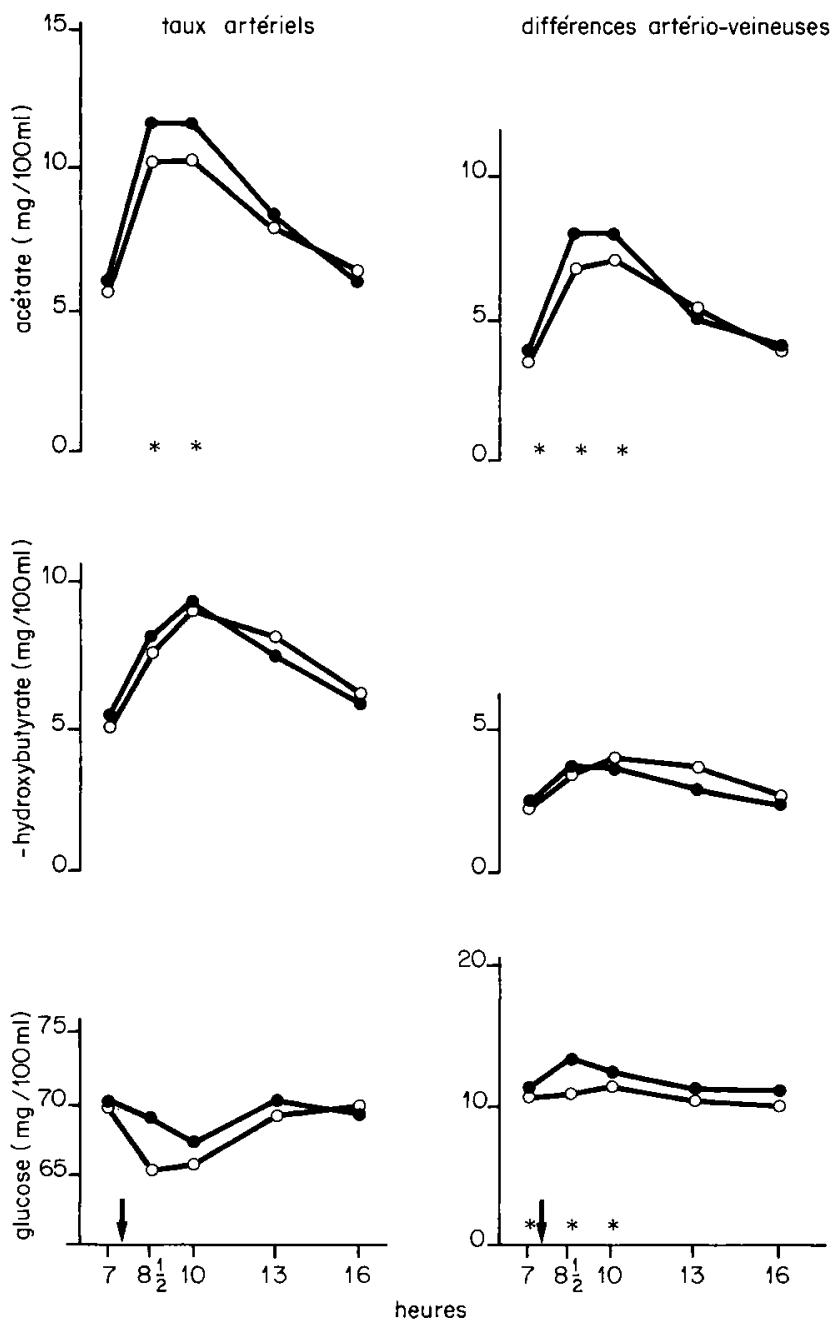

FIG. 2. - Effets du niveau azoté sur les évolutions des paramètres du métabolisme énergétique. - niveau Haut ; $O$ : niveau Bas ; I distribution des aliments ; $P<0,1$ : $P<0,05:{ }^{*}$.

ramifiés (50 à $69 \%$ ), de lysine (73\%) et de phénylalanine (25\%) sont significatives (tabl. 2). Les différences $A-V$ des acides aminés non indispensables ne sont par contre pas significativement modifiées par les variations du niveau azoté (tabl. 2).

Effets associatifs de l'énergie et de l'azote.

L'énergie et l'azote n'ont pas eu d'effets associatifs significatifs sur les paramètres du métabolisme énergétique, mais seulement sur les taux artériels d'urée $(P<0,01)$, d'isoleucine $(P<0,1)$, de valine $(P<0,05)$, de phénylalaline 
$(P<0,05)$ et de thréonine $(P<0,1)$. En effet, les augmentations consécutives à l'infusion de caséinate des taux artériels de ces métabolites sont respectivement 1,$4 ; 1,4 ; 1,5 ; 2,1$ fois plus et 3,5 fois moins élevées avec le niveau énergétique bas qu'avec le haut.

\section{Discussion.}

\section{Effets du niveau énergétique.}

La légère réduction de la sécrétion des acides gras courts et des acides gras longs du lait observée à la suite de l'accroissement du niveau énergétique par infusion ruminale d'un mélange d'AGV à forte proportion d'acide propionique (Rulquin, 1982) reflète celle des différences artério-veineuses mammaires de leurs précurseurs respectifs que sont l'acétate et le $\beta$-hỵdroxybutyrate d'une part, et les triglycérides (estimés par la mesure du glycérol total) d'autre part. Cependant, l'ampleur de la diminution des différences artério-veineuses d'acétate et de $\beta$-hydroxybutyrate est notablement plus importante que celle de la sécrétion des acides gras courts. Cette discordance pourrait être expliquée par une diminution du débit sanguin mammaire entraînée par le déficit énergétique, mais cela n'a été étudié et démontré que dans le cas du jeûne (Linzell, 1967). En raison de l'éventuelle incompétence des valvules de la veine pudique externe, il est aussi possible que le sang veineux mammaire ait été contaminé par du sang veineux non mammaire. En pratique, ce risque de contamination semble très faible (Mepham, 1982).

Pour l'acétate et le $\beta$-hydroxybutyrate, la diminution des différences artérioveineuses est une conséquence directe de la baisse des taux circulants en raison des relations étroites liant les différences artério-veineuses aux taux artériels (Williams et Elliot, 1980 ; Rulquin, 1981), alors que pour les triglycérides, elles doit probablement être attribuée à une réduction des capacités de la mamelle à les prélever dans la circulation sanguine, ou à d'éventuelles variations de la part du glycérol libre (ne représentant pas des triglycérides) dans le glycérol total.

La réduction des taux artériels d'acétate et de $\beta$-hydroxybutyrate ne semble pas provenir d'une diminution de leur production au niveau du rumen, car les variations des concentrations de leurs précurseurs (acide acétique et acide butyrique) dans le jus de rumen sont nulles (cas de l'acide butyrique) ou ne correspondent qu'à un effet de dilution venant de I'infusion d'AGV (cas de l'acide acétique) (Rulquin, 1982). Il est probable que cette réduction provienne d'un accroissement de l'utilisation de ces métabolites par les tissus extra-mammaires, comme l'ont démontré Pethick et Lindsay (1981) pour l'acétate. Cet effet est sans doute sous régulation hormonale, notamment de l'insuline dont le niveau augmente avec les régimes riches en aliments concentrés ou avec des infusions d'acide propionique dans le rumen (cf. revue de Trenkle, 1981).

La réduction de la capacité de prélèvement des triglycérides par la mamelle consécutive à l'accroissement du niveau énergétique, est similaire à celle observée avec des régimes riches en aliments concentrés (Annison, Bickerstaffe et Linzell, 1974). Elle pourrait correspondre à la diminution de l'activité de la lipoprotéine lipase mammaire qui est enregistrée avec ce type de régime (Emery, 1973). 
Contrairement à ce qui se passe pour les précurseurs des acides gras du lait, l'accroissement du niveau énergétique ne modifie pas les différences artérioveineuses des acides aminés et augmente légèrement la quantité de protéines secrétées dans le lait (Rulquin, 1982). La baisse des taux artériels des acides aminés indispensables et plus particulièrement celle des acides aminés ramifiés qu'il entraîne est similaire à celles observées à la suite d'infusion d'acide propionique (Halfpenny, Rook et Smith, 1969) ou de glucose dans le duodénum (cf. revue de Clark, 1975). Elle ne semble pas provenir d'une réduction des quantités d'acides aminés absorbés dans l'intestin (Rulquin, 1982), mais plutôt d'une diminution de leur catabolisme, comme l'indique la baisse de l'urémie dans notre essai ou l'augmentation de la rétention azotée des vaches laitières à la suite d'infusion intraruminale d'acide propionique (Orskov et al., 1969).

Chez le monogastrique, la baisse de l'aminoacidémie consécutive à l'administration de glucose serait due à un prélèvement préférentiel des acides aminés par les tissus extra-hépatiques (les muscles notamment) sous l'effet de l'insuline (Munro, 1964). II est possible que l'insuline agisse de la même manière sur les prélèvements des acides aminés effectués par la mamelle, ce qui expliquerait qu'ils se maintiennent au même niveau malgré une baisse des taux artériels.

Malgré un accroissement de la glycémie, provenant sans doute d'une augmentation de la néoglucogénèse à partir de l'acide propionique (Judson et Leng, 1973), les différences artério-veineuses de glucose ont diminué. Ces observations sont comparables à celles enregistrées avec des régimes riches en aliments concentrés par Ovcharenko et Islamova (1979), et pourraient correspondre à des effets hormonaux.

L'insuline ne paraît pas être en jeu (Laarveld, Christensen et Brockman, 1981) ; les glucocorticoïdes, dont le niveau est aussi accru avec des régimes riches en aliments concentrés (Mils et Jenny, 1979) pourraient par contre être à l'origine de ces variations. En effet, des injections d'ACTH ont provoqué des variations opposées du taux artériel et des différences artério-veineuses mammaires de glucose (Stewart et Thompson, 1981). Les glucocorticoïdes, selon Kronfeld et Hartmann (1973), modifieraient le transport du glucose à travers les parois des capillaires.

En dépit de la baisse des différences artério-veineuses de glucose, la mamelle a synthétisé autant de lactose (Rulquin, 1982) ; cela pourrait être expliqué par la variation du débit sanguin mammaire envisagée précédemment.

\section{Effets du niveau azoté.}

Les accroissements des quantités de matières grasses, de lactose et de protéines secrétées dans le lait qui résultent de l'élévation du niveau azoté (Rulquin, 1982) sont cohérents avec les augmentations des différences artério-veineuses mammaires de leurs précurseurs. Cependant, l'augmentation des différences artério-veineuses d'acétate et des triglycérides semble à elle seule insuffisante pour expliquer la totalité de l'accroissement des quantités d'acides gras synthétisés (C4 à C16) par la mamelle.

L'augmentation du taux artériel et des différences artério-veineuses d'acétate ne semble pas provenir d'un effet direct de l'infusion de caséinate mais plutôt 
d'un effet des AGV qui ont été infusés en quantité différente suivant les niveaux azotés afin de les rendre isoénergétiques.

Le supplément d'acides aminés apporté par le caséinate, contrairement à l'effet qu'il produit en début de lactation sur des vaches en déficit énergétique (Verite et Journet, 1977 ; Journet et Rémond, 1981 ; Orskov, Grubb et Kay, 1977), n'entraîne pas un accroissement important de la mobilisation des lipides corporels, comme l'indiquent les faibles variations des taux sanguins d'AGNE et de $\beta$-hydroxybutyrate et les teneurs du lait en acides gras à 18 atomes de carbone. Toutefois, il semble favoriser, par un mécanisme qui reste à étudier (effet sur la régulation de la lipoprotéine lipase ?), la captation des triglycérides sanguins par la mamelle.

Le caséinate infusé augmente la néoglucogénèse (Judson et Leng, 1973) et permet ainsi d'accroître la quantité de glucose disponible au niveau mammaire pour la synthèse du lactose et, par ce biais, la production de lait. Cependant, par comparaison à l'effet faible ou nul d'une infusion de glucose sur la production laitière, la caséine agit probablement peu par son effet glucogénique (Ranawana et Kellaway, 1977b ; Clark et al., 1977).

L'augmentation du taux des acides aminés indispensables du sang consécutive à l'infusion de caséinate a déjà été observée sur des vaches laitières (cf. revue de Clark, 1975 ; Clark et al., 1977 ; Vik-Mo et al., 1974) ou sur des chèvres laitières (Ranawana et Kellaway, 1977b ; Gow et al., 1979) ; les acides aminés ramifiés présentent toujours les plus fortes hausses. L'accroissement de leurs différences artério-veineuses mammaires qui en résulte correspond aux observations de Ranawana et Kellaway (1977a) et de Clark et al. (1977) à l'exception de l'arginine, à celles de Spires et al. (1975).

Dans cet essai, l'accroissement des différences artério-veineuses des acides aminés indispensables coïncide avec une production supplémentaire de protéines dans le lait qui, cependant, ne représente que $33 \%$ de la quantité de protéines infusées (Rulquin, 1982). Cette réponse relativement faible provient peut-être de l'existence d'un ou plusieurs acides aminés limitants. Pour préciser les acides aminés indispensables limitants, nous avons établi la relation entre les quantités entrant dans l'intestin (Rulquin, 1982) et les concentrations artérielles (fig. 3). Le classement des acides aminés en fonction du rapport entre leur ordonnée et leur abscisse pour-

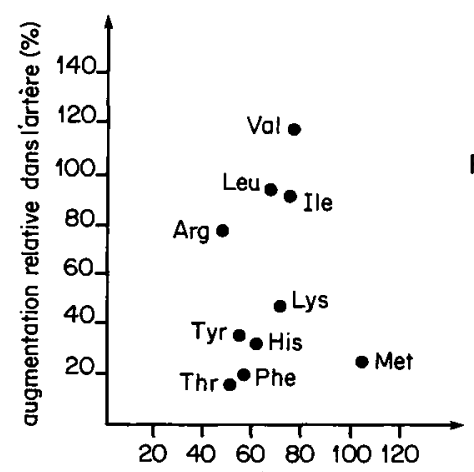

FIG. 3. - Relations entre les quantités d'acides aminés entrant dans le duodénum et leurs concentrations artérielles. (moyenne des deux niveaux énergétiques). 
rait indiquer l'ordre dans lequel ils sont susceptibles d'être limitants (plus le rapport est faible, plus l'acide aminé est limitant). II apparaît ainsi deux groupes significativement différents $(P<0,05)$ :

- les acides aminés qui ne sont probablement pas limitants, c'est-à-dire, l'arginine et les acides aminés ramifiés,

- les acides aminés qui semblent être limitants, c'est-à-dire par ordre d'importance, la méthionine, la thréonine, la phénylalanine, l'histidine, la lysine et la tyrosine.

II apparaît que, lorsque le niveau énergétique est proche des besoins (98\% des besoins), les acides aminés qui ont le plus limité la réponse à l'infusion de caséine sont la méthionine, la thréonine et la phénylalanine. Mepham (1982), par une approche différente, considère ces acides aminés ainsi que la lysine comme étant les plus limitants.

De la comparaison entre les accroissements des quantités secrétées des différents constituants du lait et ceux des différences artério-veineuses mammaires de leurs précurseurs, il ressort que vraisemblablement l'infusion de caséinate a provoqué une augmentation du débit sanguin mammaire. II est possible que cet effet se produise par l'intermédiaire de l'hormone de croissance. En effet, l'infusion post-ruminale de caséinate provoque une sécrétion d'hormone de croissance (Barry, 1980 ; Oldham, 1981). Or, l'injection de cette hormone accroît le débit sanguin (cf. Tucker, 1981 ; Mepham, 1982).

\section{Effets associatifs de l'azote et de l'énergie.}

Le supplément d'acides aminés apportés par l'infusion de caséinate semble participer davantage à l'anabolisme protéique, notamment au niveau mammaire (Rulquin, 1982) lorsque le niveau énergétique est élevé, que lorsqu'il est bas. Au niveau du sang, cela se traduit par une baisse des concentrations des acides aminés et plus particulièrement de ceux (valine et isoleucine) qui ont tendance à s'accumuler lorsque le niveau énergétique est bas (fig. 4). En comparant, comme précédemment, les variations relatives dans l'artère et dans le duodénum, il apparaît que la phénylalanine, la thréonine et la méthionine sont limitantes quel que
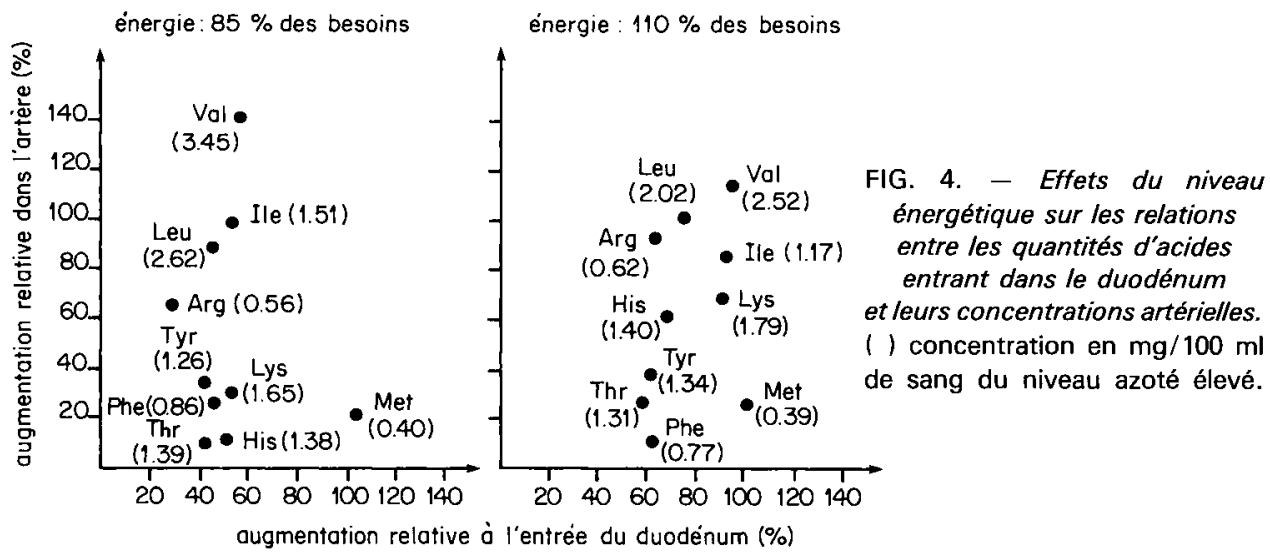

augmentation relative à l'entrée du duodénum (\%) 
soit le niveau énergétique, alors que l'histidine, et peut-être la lysine, ne sont limitants qu'en cas de déficit énergétique.

II est possible que, l'effet glucogénique de la caséine s'ajoutant à celui de l'acide propionique, la production endogène de glucose soit supérieure avec le niveau haut permettant ainsi une synthèse de lactose plus élevée (Rulquin, 1982).

Contrairement aux résultats d'Ørskov, Reid et Mc Donald (1981), la mobilisation des réserves corporelles ne semble pas être en cause dans cette interaction des apports énergétiques et azotés car aucun des paramètres ( $\beta$-hydroxybutyrate, AGNE, acides gras longs du lait) susceptibles de révéler une telle mobilisation n'ont été affectés.

\section{Conclusion.}

Les variations de la synthèse des constituants du lait engendrées par des modifications du rapport des produits terminaux de la digestion peuvent s'interpréter à l'aide des mesures des différences artério-veineuses mammaires de leurs précurseurs. Toutefois, la mesure du débit sanguin mammaire s'avère nécessaire pour pouvoir expliquer ces variations, et ce d'autant plus que le débit sanguin est susceptible de varier lui aussi en fonction du rapport des produits terminaux de la digestion. Les résultats de cet essai et les données bibliographiques permettent de penser que :

$1^{\circ} L^{\prime}$ acide propionique réduit la synthèse des constituants du lait de deux manières:

- d'une part, en favorisant, probablement par l'intermédiaire de l'insuline, la captation des nutriments (acétate, $\beta$-hydroxybutyrate, acides aminés) par les tissus adipeux et les muscles, il réduit la quantité de nutriments qui arrivent à la mamelle ;

- d'autre part, il réduit le degré de captation de certains nutriments (glucose et triglycérides) par la mamelle.

$2^{\circ}$ Un apport supplémentaire d'acides aminés augmente la synthèse des constituants du lait en accroissant la quantité de nutriments qui arrivent à la mamelle. Cet effet semble provenir d'une amélioration de l'utilisation de l'azote pour les synthèses protéiques et la synthèse du glucose. Cette amélioration est d'autant plus grande que le niveau énergétique est élevé et que la forme d'énergie est glucoformatrice.

Reçu en mars 1982.

Accepté en août 1983.

Remerciements. - Nous remercions vivement $M$. Lefaivre pour la réalisation des opérations chirurgicales, M. Pion et ses collaborateurs pour les dosages d'acides aminés et $M$. Bas pour le dosage du glycérol. 


\section{Références}

ANNISON E. F., BICKERSTAFFE R., LINZELL J. L., 1974. Glucose and Fatty acid metabolism in cows producing milk of low fat content. J. agric. Sci. Camb., 82, 87-95.

ANTONIS A., 1965. Semiautomated method for the colorimetric determination of plasma free fatty acids. J. Lipid Res., 6, 307-312.

BARRY T. N., 1980. Responses to abomasal infusions of casein plus methionine in lactating ewes fed fresh pasture. N. Z. J. agric. Res., 23, 427-431.

CLARK J. H., 1975. Lactational responses to postruminal administration of proteins and amino acids. J. Dairy Sci., 58, 1178-1197.

CLARK J. H., SPIRES H. R., DERRIG R. G., BENNINK M. R., 1977. Milk production, nitrogen utilization and glucose synthesis in lactating cows infused postruminally with sodium caseinate and glucose. J. Nutr., 107, 631-644.

COCHRAN W. G., COX G. M., 1968. Incomplete latin squares, 507-544. In COCHRAN W. G., COX G. M., Experimental designs. John Wiley and Sons, New-York.

EMERY R. S., 1973. Biosynthesis of milk fat. J. Dairy Sci., 56, 1187-1195.

GORDON F. J., FORBES T. J., 1970. The associative effect of energy and protein intake in the dairy cow. J. Dairy Res., 37, 481-491.

GOW C. B., RANAWANA S. S. E., KELLAWAY R. C., Mc DOWELL G. H., 1979. Responses to post-ruminal infusion of casein and arginine, and to dietary protein supplements in lactating goats. Br. J. Nutr., 41, 371-382.

HALFPENNY A. F., ROOK J. A. F., SMITH G. H., 1969. Variations with energy nutrition in the concentrations of amino-acids of the blood plasma in the dairy cow. Br. J. Nutr., 23, 547557.

JOURNET M., RÉMOND B., 1981, Response of dairy cow to protein level in early lactation. Livestock Product. Sci., 8, 21-35.

JUDSON G. J., LENG R. A., 1973. Studies on the control of gluconeogenesis in sheep: effect of proprionate, casein and butyrate infusions. Br. J. Nutr., 29, 175-195.

KRONFELD D. S., HARTMANN P. E., 1973. Glucose redistribution in lactating cow given dexamethasone. J. Dairy Sci., 56, 903-908.

LAARVELD B., CHRISTENSEN D. A., BROCKMAN R. P., 1981. The effect of insulin on net metabolism of glucose and amino acids by the bovine mammary gland. Endocrinology, 108, 2217-2221.

LINZELL J. L., 1967. The effect of very frequent milking and of oxytocin on the yield and composition of milk in fed and fasted goats. J. Physiol., 190, 333-346.

MEPHAM T. B., 1982. Amino acid utilization by lactating mammary gland. J. Dairy Sci., 65, $287-$ 298.

MILLS S. E., JENNY B. F., 1979. Effects of high concentrate feeding and fasting on plasma glucocorticoids in dairy heifers. J. anim. Sci., 48, 961-965.

MUNRO H. N., 1964. General aspects of the regulation of protein metabolism by diet and hormones, 381-481. In MUNRO H. N., ALLISON J. B., Vol. I, Mammalian protein metabolism, Acad. Press, New-York.

OLDHAM J. D., 1981. Amino acid requirements for lactation in high-yielding dairy cows, $49-81$. In HARESIGN W., COLE D. J. A., Recent developments in ruminant nutrition. Butterworths.

ØRSKOV E. R., FLATT W. P., MOE P. W., MUNSON A. W., HEMKEN R. W., KATZ J., 1969 The influence of ruminal infusion of volatile fatty acids on milk yield and composition and on energy utilization by lactating cows. Br. J. Nutr., 23, 443-453.

ØRSKOV E. R., GRUBB D. A., KAY R. N. B., 1977. Effect of postruminal glucose or protein supplementation on milk yield and composition in Friesian cows in early lactation and negative energy balance. Br. J. Nutr., 38, 397-405.

$\emptyset$ RSKOV E. R., REID G. W., Mc DONALD I., 1981. The effects of protein degradability and food intake on milk yield and composition in cows in early lactation. Br. J. Nutr., 45, 547-555.

OVCHARENKO E. V., ISLAMOVA N. I., 1979. Absorption by the udder of milk fat precursors in cows kept on fat-depressing rations. Dairy Sci. Abstr., 41, Abst. 1181.

PAWLAK M., PION R., 1968. Influence de la supplémentation des protéines de blé par des doses croissantes de lysine sur la teneur en acides aminés libres du sang et du muscle de rat en croissance. Ann. Biol. anim. Bioch. Biophy., 8, 517-530.

PETHICK D. W., LINDSAY D. B., 1981. The low milk-fat syndrome - a preference for muscle over milk. In GIESECKE D., DIRKSEN G., STANGASSINGER H., Metabolic disorders in farm animals. Institut für Physiologie, Physiologische Chemie und Ernährungsphysiologie, Tierärztliche Fakultät der Universität Munchen. 
RANAWANA S. S. E., KELLAWAY R. C., 1977a. Response to postruminal infusions of graded level of casein in lactating goats. Br. J. Nutr., 37, 67-79.

RANAWANA S. S. E., KELLAWAY R. C., 1977b. Response to postruminal infusions of glucose and casein in lactating goats. Br. J. Nutr., 37, 395-402.

RULOUIN H., 1981. Etude méthodologique sur la mesure des différences artério-veineuses mammaires chez la vache laitière. Reprod. Nutr. Dévelop., 21, 31-46.

RULQUIN H., 1982. Effets sur la digestion et le métabolisme des vaches laitières d'infusions d'acides gras volatils dans le rumen et de caséinate dans le duodénum. I. Production et digestion. Reprod. Nutr. Dévelop., 22, 905-921.

SPIRES H. R., CLARK J. H., DERRIG R. G., DAVIS C. L., 1975. Milk production and nitrogen utilization in response to postruminal infusion of sodium caseinate in lactating cows. J. Nutr., 105, 1111-1121.

STEWART H. J., THOMPSON G. E., 1981. Mammary secretion of lactose independent of glucose uptake following ACTH treatments of goats in late lactation : an in vivo study. $J$. Physiol., 319, 93 P.

STORRY J. E., ROOK J. A. F., 1965. Effect in the cow of intraruminal infusions of volatile fatty acids and of lactic acid on the secretion of the component fatty acids of the milk fat and on the composition of blood. Biochem. J., 96, 210-217.

TRENKLE A., 1981. Endocrine regulation of energy metabolism in ruminants. Fed. Proc., 40, 25362541 .

TUCKER A. H., 1981. Physiological control of mammary growth lactogenesis and lactation. J. Dairy Sci., 64, 1403-1421.

VERITE R., JOURNET M., 1977. Utilisation des tourteaux traités au formol par les vaches laitières. II. - Effets sur la production laitière du traitement des tourteaux et du niveau d'apport azoté au début de la lactation. Ann. Zootech., 26, 183-205.

VIK-MO L., HUBER J. T., BERGEN W. G., LICHTENWALNER R. E., EMERY R. S., 1974. Blood metabolites in cows abomasally infused with casein or glucose. J. Dairy Sci., 57, 1024-1030.

WILLIAMS J. A., ELLIOT J. M., 1980. Mammary extraction of propionate in lactating cows. $J$. Dairy Sci., 63, 1999-2005. 